\title{
Transcranial Direct Current Stimulation for the Treatment of Focal Hand Dystonia
}

\author{
David H. Benninger, MD, ${ }^{1,2 \star}$ Mikhail Lomarev, MD, ${ }^{1,3}$ Grisel Lopez, MD, ${ }^{1}$ Natassja Pal, ${ }^{1}$ David A. Luckenbaugh, ${ }^{4}$ \\ and Mark Hallett, $\mathrm{MD}^{1}$ \\ ${ }^{1}$ Medical Neurology Branch, National Institutes of Health, Bethesda, Maryland, USA \\ ${ }^{2}$ Department of Neurology, University Hospital of Basel, Basel, Switzerland \\ ${ }^{3}$ St. Petersburg V.M. Bekhterev Psychoneurological Research Institute, Saint-Petersburg, Russia \\ ${ }^{4}$ National Institute of Neurological Disorders and Stroke and National Institute of Mental Health, National Institutes of Health, \\ Bethesda, Maryland, USA
}

\begin{abstract}
The treatment of writer's cramp, a task-specific focal hand dystonia, needs new approaches. A deficiency of inhibition in the motor cortex might cause writer's cramp. Transcranial direct current stimulation modulates cortical excitability and may provide a therapeutic alternative. In this randomized, double-blind, sham-controlled study, we investigated the efficacy of cathodal stimulation of the contralateral motor cortex in 3 sessions in 1 week. Assessment over a 2-week period included clinical scales, subjective ratings, kinematic handwriting analysis, and neurophysiological evaluation. Twelve patients with unilateral dystonic writer's cramp were investigated; 6 received transcranial direct current
\end{abstract}

and 6 sham stimulation. Cathodal transcranial direct current stimulation had no favorable effects on clinical scales and failed to restore normal handwriting kinematics and cortical inhibition. Subjective worsening remained unexplained, leading to premature study termination. Repeated sessions of cathodal transcranial direct current stimulation of the motor cortex yielded no favorable results supporting a therapeutic potential in writer's cramp. (C) 2011 Movement Disorder Society

Key Words: transcranial direct current stimulation (tDCS); noninvasive brain stimulation; therapeutic study; focal hand dystonia
Writer's cramp (WC) is a task-specific focal hand dystonia (FHD) characterized by involuntary muscle spasms, overflow of activity including excessive activity of antagonist muscles, and impaired voluntary motor control during writing. The pathophysiology remains incompletely understood. Evidence points to a deficiency of inhibitory circuits, particularly of the motor cortex.

WC represents a therapeutic challenge. Promising results of deep brain stimulation (DBS) in dystonia

\footnotetext{
*Correspondence to: Dr. David H. Benninger, MD, Department of Neurology, Centre Hospitalier Universitaire Vaudois (CHUV), Rue du Bugnon 46, 1011 Lausanne, Switzerland; David.Benninger@chuv.ch Relevant conflicts of interest/financial disclosures: Nothing to report. This research was supported by the Intramural Research Program of the NINDS, NIH.

Full financial disclosures and author roles may be found in the online version of this article.
}

Received: 22 October 2010; Revised: 28 December 2010; Accepted: 1 February 2011

Published online in Wiley Online Library (wileyonlinelibrary.com). DOI: $10.1002 / \mathrm{mds} .23691$ and noninvasive brain stimulation, foremost repetitive transcranial magnetic stimulation (rTMS) in WC,,$^{1,2}$ indicate their therapeutic potential.

In transcranial direct current stimulation (tDCS), a direct current is continuously applied by surface electrodes on the head, which contrasts with the electric impulse induced by the short-lasting magnetic field in TMS. The possibility of modulating cortical excitability ${ }^{3,4}$ and promoting motor learning in healthy individuals ${ }^{5}$ and functional improvement in Parkinson's disease ${ }^{6}$ and in stroke $^{7}$ has raised interest in tDCS as an intervention in various brain disorders. Cathodal tDCS decreases excitability of the motor cortex $\mathrm{x}^{3,4}$ and might act on the pathophysiology in WC by compensating for the postulated deficiency in inhibition. Successful trials of low-frequency rTMS $^{1,2}$ that is inhibitory suggest a similar mechanism.

In this double-blind, randomized, sham-controlled study, we investigated whether cathodal tDCS of the motor cortex improves WC. 


\section{Patients and Methods}

Adults with primary WC were included. Exclusion criteria were bilateral WC, secondary causes of FHD, generalized dystonia, significant illnesses, pregnancy, epilepsy, substance abuse, metal devices in the head, and botulinum toxin injection within 10 weeks.

We applied tDCS in 3 sessions in the first study week (Monday, Wednesday, Friday) without concurrent intervention. A battery-driven stimulator, Phoresor II Model PM850 (IOMED, Salt Lake City, UT), delivered tDCS through electrodes. Random assignment to tDCS or sham stimulation was computer generated.

In the tDCS treatment group, cathodal tDCS $(2 \mathrm{~mA})$ was delivered for 20 minutes through $3 \times 3 \mathrm{~cm}$ electrodes (current density, $0.22 \mathrm{~mA} / \mathrm{cm}^{2}$ ). We placed the cathode on the primary motor cortex (M1) at the optimal position for motor-evoked potentials (MEPs) in the primarily affected hand muscle(s) that we determined with TMS (Magstim200 TMS machine; Whitland, Dyfed, UK) with a figure-of-eight $(70-\mathrm{mm})$ coil and the anode on the contralateral mastoid. In the sham condition, we applied DC current $(1 \mathrm{~mA})$ for 12 minutes with the anode and cathode over the forehead, short-circuited through the skin, generating the same temporary "tingling" without effects on the brain. We set up stimulation out of sight of patients and blinded investigators.

Assessment included clinical scales, subjective ratings, kinematic handwriting analysis, and neurophysiological evaluation of cortical inhibition at baseline, immediately, and a week after the last intervention.

The blinded rater evaluated the severity of WC applying Fahn's Arm Dystonia Disability Scale $(\mathrm{ADDS})^{8}$ and the WC Rating Scale (WCRS). ${ }^{9}$ In the ADDS, difficulty in various hand activities is scored, and the total score quotient is subtracted from $100 \%$, representing normal function. ${ }^{8}$ The WCRS quantifies WC during handwriting, increasing with severity. ${ }^{9}$ The primary outcome measure was the change in disability (ADDS) a week after the last intervention.

After each intervention, patients rated handwriting using a verbal scale with 4 levels ranging from 0 (none) to 3 (major improvement) and a visual analog scale (VAS) ranging from $0 \%$ (none) to $100 \%$ (full recovery).

Kinematic analysis of handwriting was computed. ${ }^{10,11}$ Patients wrote for 20 seconds on a digitizing tablet (WACOM Intuos A4, $430 \times 300 \mathrm{~mm}$ ) with high spatial $(0.05 \mathrm{~mm})$ and temporal resolution (200$\mathrm{Hz}$ sampling rate). The program computes the kinematic profile from velocity and acceleration of stylus movement in the $\mathrm{x} / \mathrm{y}$ directions. ${ }^{12}$ The following kinematic variables discriminated handwriting in WC from controls (unpublished) Mean positive stroke duration (time for an upward stroke), the number of inversions in velocity (NIV) and acceleration (NIA; number of velocity and acceleration peaks during a single stroke that inversely measures smoothness of handwriting), and coefficient of variation $(\mathrm{CV})$ of mean peak velocity (measures variability in the velocity profile of consecutive strokes) were increased in WC, and frequency of strokes and percentage of strokes with number of inversions equal to 1 (measures handwriting consistency) were decreased. In addition, velocity was included. We discarded the measurement of vertical pressure on the tablet because the recordable maximum of 4 newtons was often exceeded. This invalidated the movement score composed of kinematic variables including pressure, which was the initial primary outcome measure.

We determined short intracortical inhibition (SICI) in the motor cortex in a paired-pulse paradigm with a 3-ms interstimulus interval 1 (ISI) between subthreshold conditioning $(80 \%$ rest motor threshold [RMT]) and the suprathreshold test TMS pulse (120\% RMT) targeting M1 area of the primarily affected muscle. ${ }^{13}$ We calculated SICI as the ratio of paired pulse to test MEP amplitudes. We determined RMT to the nearest $1 \%$ of the stimulator output required to elicit an MEP of $\geq 50 \mu \mathrm{V}$ in $\geq 5$ of 10 trials.

The NIH Institutional Review Board approved the registered study (ClinicalTrials.gov: NCT00106782) and its premature termination. All participants gave written informed consent.

\section{Statistical Analysis}

Full-factorial repeated-measures analysis of variance (ANOVA) was applied with between-subjects factor for treatment (Treatment) and within-subjects factor for time (Time). Omnibus main effects and interactions were examined post hoc using Bonferroniadjusted simple effects tests within the context of ANOVA-ANCOVA. A priori comparisons were made as specified. Levene's test was used to verify the homogeneity of variance assumption, and Shapiro-Wilk's test and standardized residuals were examined to verify the normality assumption. The independent $t$ test or Wilcoxon rank sum test and Fisher's exact test, whichever appropriate, were applied to compare groups on demographic and clinical findings. Significance was evaluated at $P<.05,2$-tailed. Bonferroni's procedure corrected for multiple comparisons. We used SPSS, version 17.0.1.

\section{Results}

We performed an interim analysis because 2 patients receiving $\mathrm{tDCS}$ reported short-lasting worsening of handwriting after their last intervention, but we could not substantiate clinical deterioration or changes in 


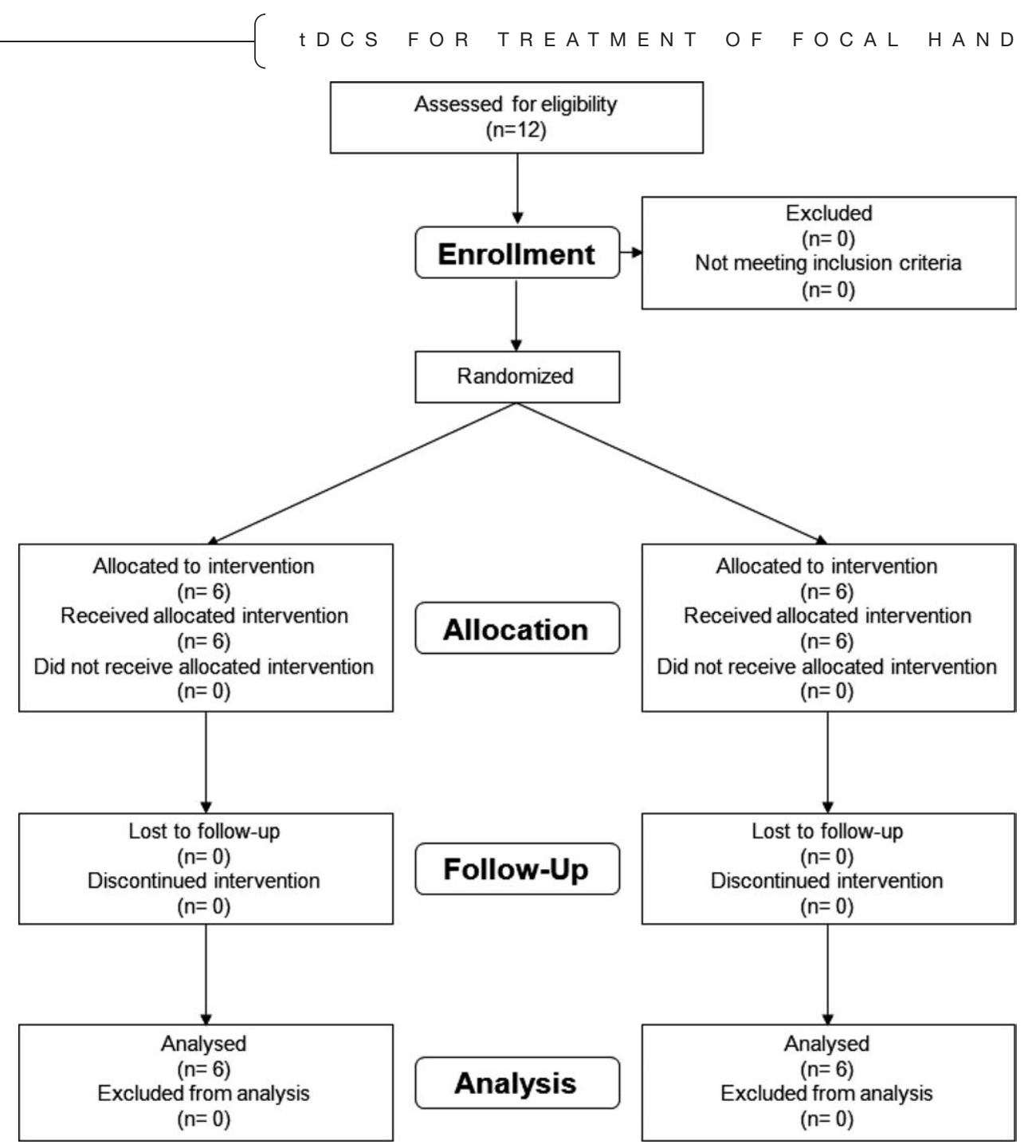

FIG. 1. Flow diagram of patients with writer's cramp (WC) enrolled in this therapeutic study (http://www.consort-statement.org).

kinematics and neurophysiology. Because deleterious effects of cathodal stimulation could not be excluded and preliminary results were not favorable, we opted to terminate the study. We had enrolled 12 patients $(4$ women; mean age, $57.1 \pm 6.6$ years; range, 44-67 years) with dystonic WC (mean duration, $13.3 \pm 5.9$ years; range, 4-20 years) of the dominant hand (all right-handed but 1), randomly assigned to tDCS ( $\mathrm{n}=$ 6) or sham stimulation ( $\mathrm{n}=6$; Fig. 1). Demographics and clinical findings at baseline did not differ between groups. No patient was taking drugs for WC. All had received botulinum -toxin, but only 7 responded.

Cathodal tDCS had no effects on disability (ADDS) and severity of WC (WCRS; Table 1). Even more, those receiving sham stimulation reported more improvement than those with tDCS in the verbal scale $(1.1 \pm 0.27$ vs $0.3 \pm 0.3, P=.001)$, whereas not significantly in the VAS $(22.8 \pm 11.4$ vs $14.0 \pm 14.2$, $P=.26)$.

Cathodal tDCS had no effects on kinematics, but we found a trend to a decrease in NIV in the sham group $(P=.051$, uncorrected $)$ indicating smoother handwrit- ing. Cathodal tDCS did not modulate short intracortical inhibition. All experienced short-lasting "tingling" but no pain or other adverse events.

\section{Discussion}

In this controlled study, cathodal stimulation of the motor cortex yielded no favorable effects on disability and severity of dystonic WC and failed to restore normal kinematics of handwriting and cortical inhibition. Sham-stimulated patients even reported significantly better improvement contrasting with the subjective worsening with tDCS, which remained unexplained and constituted the reason for the premature study termination. These findings are consistent with the lack of beneficial effects of a single session of cathodal tDCS on FHD in guitarists ${ }^{14}$ and extend knowledge by demonstrating ineffectiveness of repeated interventions.

There is evidence for abnormal plasticity considered maladaptive in dystonia contributing to the pathogenesis. This could explain the progressive development and 
B E N N I N GR E T A L .

TABLE 1. Clinical scales, kinematics of handwriting, and neurophysiology at baseline (mean \pm standard error), immediately after, and 1 week after the last tDCS or sham intervention (adjusted mean \pm standard error)

\begin{tabular}{|c|c|c|c|c|c|c|}
\hline & tDCS sham & Baseline & After last intervention & $P$ & 1 Week after last intervention & $P$ \\
\hline \multicolumn{7}{|l|}{ Clinical scales } \\
\hline \multirow[t]{2}{*}{ ADDS } & & $52.5 \pm 8.9$ & $59.8 \pm 4.1$ & 0.67 & $59.6 \pm 2.0$ & 0.14 \\
\hline & & $66.0 \pm 7.9$ & $62.4 \pm 4.1$ & & $64.2 \pm 2.0$ & \\
\hline \multirow[t]{2}{*}{ WCRS } & & $9.3 \pm 1.7$ & $11.5 \pm 1.1$ & 0.38 & $10.8 \pm 1.3$ & 0.38 \\
\hline & & $14.0 \pm 2.7$ & $10.0 \pm 1.0$ & & $9.1 \pm 1.1$ & \\
\hline \multicolumn{7}{|l|}{ Kinematics of handwriting } \\
\hline \multirow[t]{2}{*}{ Frequency of strokes $(\mathrm{Hz})$} & & $3.4 \pm 0.14$ & $3.3 \pm 0.17$ & 0.4 & $3.6 \pm 0.19$ & 0.24 \\
\hline & & $3.0 \pm 0.14$ & $3.1 \pm 0.17$ & & $3.3 \pm 0.19$ & \\
\hline \multirow[t]{2}{*}{ NIV } & & $1.22 \pm 0.08$ & $1.24 \pm 0.06$ & 0.13 & $1.21 \pm 0.04$ & 0.051 \\
\hline & & $1.42 \pm 0.08$ & $1.37 \pm 0.06$ & & $1.35 \pm 0.04$ & \\
\hline \multirow[t]{2}{*}{ Velocity (mm/s) } & & $62.2 \pm 4.8$ & $63.6 \pm 6.1$ & 0.74 & $70.1 \pm 6.2$ & 0.55 \\
\hline & & $55.9 \pm 4.8$ & $66.6 \pm 6.1$ & & $64.5 \pm 6.2$ & \\
\hline \multirow[t]{2}{*}{ Positive stroke duration (ms) } & & $150.5 \pm 9.3$ & $160.5 \pm 14.3$ & 0.82 & $155.6 \pm 13.3$ & 0.87 \\
\hline & & $157.6 \pm 9.3$ & $165.4 \pm 14.3$ & & $158.6 \pm 13.3$ & \\
\hline \multirow[t]{2}{*}{ NIA } & & $2.05 \pm 0.11$ & $2.09 \pm 0.15$ & 0.49 & $1.87 \pm 0.14$ & 0.30 \\
\hline & & $2.14 \pm 0.11$ & $2.25 \pm 0.15$ & & $2.09 \pm 0.14$ & \\
\hline \multirow[t]{2}{*}{ CV of peak velocity } & & $0.493 \pm 0.045$ & $0.423 \pm 0.034$ & 0.98 & $0.435 \pm 0.026$ & 0.99 \\
\hline & & $0.448 \pm 0.045$ & $0.420 \pm 0.034$ & & $0.436 \pm 0.026$ & \\
\hline \multirow[t]{2}{*}{ NIO $(\%$} & & $81.6 \pm 3.9$ & $80.3 \pm 4.3$ & 0.96 & $82.8 \pm 3.6$ & 0.65 \\
\hline & & $78.6 \pm 3.9$ & $80.6 \pm 4.3$ & & $80.3 \pm 3.6$ & \\
\hline \multicolumn{7}{|l|}{ Neurophysiology } \\
\hline \multirow[t]{2}{*}{ Inhibition (SICl; \%) } & & $54.7 \pm 10.2$ & $57.7 \pm 10.2$ & 0.73 & $60.7 \pm 4.6$ & 0.53 \\
\hline & & $66.4 \pm 11.2$ & $63.3 \pm 11.5$ & & $65.3 \pm 5.1$ & \\
\hline
\end{tabular}

tDCS group, upper value; sham group, lower value.

ADDS, Arm Dystonia Disability Scale (Fahn, 1989); CV, coefficient of variation; NIA, number of inversions in the acceleration profile; NIO, percentage of strokes with number of inversions equal to 1; NIV, number of inversions in the velocity profile SICI, short intracortical inhibition; WCRS, Writer's Cramp Rating Scale (Wissel, 1996).

the protracted improvement with DBS, along with changes in physiology over months. ${ }^{15}$ Therefore, prolonged stimulation may be required to induce effects. These could be adverse with cathodal stimulation, which needs to be excluded prior to future trials. Prolonged controlled trials are feasible with newer programmable DC stimulators allowing interventions at home.

The maladaptive plasticity in WC could cause irreversible changes and might have impeded the efficacy of tDCS, as the failure of inhibitory cathodal stimulation to decrease cortical excitability suggests. ${ }^{16}$ The variable response of WC to botulinum toxin may reflect this heterogeneity in pathophysiology, which remains incompletely understood. Structural abnormalities in the sensorimotor cortex ${ }^{17}$ could underlie maladaptive plasticity and explain why stimulatory interventions fail. These structural ${ }^{17}$ and functional ${ }^{18}$ abnormalities in the primary motor cortex provided rationale for targeting by noninvasive brain stimulation with beneficial effects. Inhibitory $1-\mathrm{Hz}$ rTMS of motor $^{1}$ and $0.2-\mathrm{Hz}$ rTMS of premotor cortex ${ }^{2}$ reportedly restored intracortical inhibition and reduced impairment. The discrepancy in therapeutic efficacy could result from differences in mechanisms of action. In contrast with pulsed stimulation in rTMS, continuous direct current of tDCS modulates membrane excitability and induces shifts in cortical excitability.
However, the increase in cortical excitability with anodal stimulation does not generate action potentials, ${ }^{3,4}$ whereas cathodal stimulation decreases excitability. ${ }^{3}$ The mechanism of action beyond immediate physiological effects remains unknown. There is evidence to suggest that tDCS spreads widely beyond stimulation site with effects in the premotor cortex areas and basal ganglia, ${ }^{19}$ where functional abnormalities are found. ${ }^{18}$

The sample size was small, but the absence of changes in kinematics and neurophysiology, which represent complementary aspects of $\mathrm{WC}^{20}$ argues against a therapeutic potential of the present cathodal tDCS protocol. Anodal or other patterns of DC stimulation and possibly longer-lasting treatment might be more potent. A promising approach could be combining tDCS with behavioral therapy. Behavioral therapies in $\mathrm{FHD}^{12,21}$ were found to be effective for regaining fine motor control and involve relearning writing and fine hand movements. Because anodal tDCS enhances motor learning in healthy individuals ${ }^{5}$ and in various brain disorders including $\mathrm{PD}^{6}$ and stroke, ${ }^{7}$ a combination of tDCS and behavioral therapy could carry therapeutic potential.

Acknowledgment: We thank David Bates for help in the research. 


\section{References}

1. Siebner HR, Tormos JM, Ceballos-Baumann AO, et al. Low-frequency repetitive transcranial magnetic stimulation of the motor cortex in writer's cramp. Neurology. 1999;52:529-537.

2. Murase N, Rothwell JC, Kaji R, et al. Subthreshold low-frequency repetitive transcranial magnetic stimulation over the premotor cortex modulates writer's cramp. Brain. 2005;128:104-115.

3. Nitsche MA, Paulus W. Excitability changes induced in the human motor cortex by weak transcranial direct current stimulation. J Physiol. 2000;527:633-639.

4. Priori A, Berardelli A, Rona S, Accornero N, Manfredi M. Polarization of the human motor cortex through the scalp. Neuroreport. 1998;9:2257-2260.

5. Nitsche MA, Schauenburg A, Lang N, et al. Facilitation of implicit motor learning by weak transcranial direct current stimulation of the primary motor cortex in the human. J Cogn Neurosci. 2003; 15:619-626.

6. Benninger DH, Lomarev M, Lopez G, et al. Transcranial direct current stimulation for the treatment of Parkinson's disease. J Neurol Neurosurg Psychiatry. 2010;81:1105-1111.

7. Hummel F, Celnik P, Giraux P, et al. Effects of non-invasive cortical stimulation on skilled motor function in chronic stroke. Brain. 2005;128:490-499.

8. Fahn S. Assessment of primary dystonia. In:Munsat TL, ed. Quantification of Neurologic Deficit. Stoneham, MA: Butterworths; 1989;241-270.

9. Wissel J, Kabus C, Wenzel R, et al. Botulinum toxin in writer's cramp: objective response evaluation in 31 patients. J Neurol Neurosurg Psychiatry. 1996;61:172-175.

10. Marquardt C, Mai N. A computational procedure for movement analysis in handwriting. J Neurosci Methods. 1994;52:39-45.
11. Marquardt C, Mai N. CS Version 5.0 Operating Manual Computational Analysis of Handwriting Movements. München, Germany; 1997.

12. Zeuner KE, Shill HA, Sohn YH, et al. Motor training as treatment in focal hand dystonia. Mov Disord. 2005;20:335-341.

13. Stinear CM, Byblow WD. Elevated threshold for intracortical inhibition in focal hand dystonia. Mov Disord. 2004;19:1312-1317.

14. Buttkus F, Weidenmuller M, Schneider S, et al. Failure of cathodal direct current stimulation to improve fine motor control in musician's dystonia. Mov Disord. 2010;25:389-394.

15. Tisch S, Rothwell JC, Limousin P, Hariz MI, Corcos DM. The physiological effects of pallidal deep brain stimulation in dystonia. IEEE Trans Neural Syst Rehabil Eng. 2007;15:166-172.

16. Quartarone A, Rizzo V, Bagnato S, et al. Homeostatic-like plasticity of the primary motor hand area is impaired in focal hand dystonia. Brain. 2005;128:1943-1950.

17. Garraux G, Bauer A, Hanakawa T, Wu T, Kansaku K, Hallett M. Changes in brain anatomy in focal hand dystonia. Ann Neurol. 2004;55:736-739.

18. Hallett M. Dystonia: abnormal movements result from loss of inhibition. In: Fahn S, Hallett M, DeLong M, eds. Dystonia 4. Advances in Neurology. Vol. 94. Philadelphia, PA: Lippincott Williams \& Wilkins; 2004:1-9.

19. Lang N, Siebner HR, Ward NS, et al. How does transcranial DC stimulation of the primary motor cortex alter regional neuronal activity in the human brain? Eur J Neurosci. 2005;22:495-504.

20. Zeuner KE, Peller M, Knutzen A, et al. How to assess motor impairment in writer's cramp. Mov Disord. 2007;22:1102-1109.

21. Candia V, Schafer T, Taub E, et al. Sensory motor retuning: A behavioral treatment for focal hand dystonia of pianists and guitarists. Arch Phys Med Rehabil. 2002;83:1342-1348. 